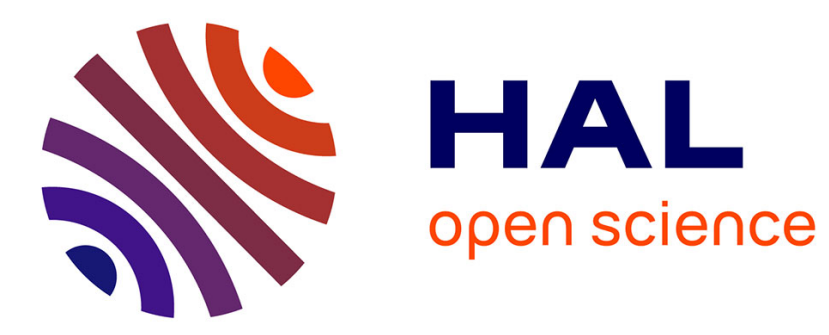

\title{
Coupling of vibro-acoustic waves with premixed flame
} Basile Radisson, Juliette Piketty-Moine, Christophe Almarcha

\section{To cite this version:}

Basile Radisson, Juliette Piketty-Moine, Christophe Almarcha. Coupling of vibro-acoustic waves with premixed flame. Physical Review Fluids, 2019, 4 (12), 10.1103/PhysRevFluids.4.121201 . hal02496062

\section{HAL Id: hal-02496062 \\ https://hal.science/hal-02496062}

Submitted on 20 May 2020

HAL is a multi-disciplinary open access archive for the deposit and dissemination of scientific research documents, whether they are published or not. The documents may come from teaching and research institutions in France or abroad, or from public or private research centers.
L'archive ouverte pluridisciplinaire HAL, est destinée au dépôt et à la diffusion de documents scientifiques de niveau recherche, publiés ou non, émanant des établissements d'enseignement et de recherche français ou étrangers, des laboratoires publics ou privés. 


\section{Coupling of vibro-acoustic waves with premixed flame}

Basile Radisson, Juliette Piketty-Moine and Christophe Almarcha Aix Marseille Université, CNRS, Centrale Marseille, IRPHE UMR 7342, 13384 Marseille, France (Dated: September 3, 2019)

We investigate the coupling between a premixed flame freely propagating inside a Hele-Shaw burner and the mechanical vibrations of the burner structure. The combustion chamber deformations are not only able to damp the classical thermoacoustic instabilities but they can also trigger a new oscillating combustion instability. We demonstrate that the flow oscillations induced by the burner vibrations can be used to control the shape of the flame surface, by damping the DarrieusLandau dynamics, or by triggering Faraday-like waves. 


\section{INTRODUCTION}

Thermo-acoustic instabilities are acoustic oscillations that arise when a flame is coupled to an acoustic mode of a combustion chamber. They are highly debated in the combustion community because of the dramatic consequences they can have on industrial burners [1], rocket engines [2], or ramjet engines [3] to name a few. In order to control these instabilities, it is of prime importance to understand the diverse phenomena at play [4-6] which participate to the necessary condition summed up in the Rayleigh [7] criterion: pressure and heat release variations have to be in phase for the acoustic instability to develop. For propagating flames, a canonical experiment was designed in vertical tubes [8] and highlights the namely first and secondary thermo-acoustic instabilities, that have an influence on the flame topology in addition to the emission of acoustic waves. During the primary instability, an increasing acoustic field flattens Darrieus-Landau self-wrinkled flames by parametric restabilization. During the secondary instability, the more intense acoustic field generates new small scale wrinkles through a Faraday like mechanism: the parametric resonance. In both cases, the fundamental acoustic mode of the tube is the most likely excited (i.e. with a wavelength four times larger than the tube length). The interaction mechanism between acoustic waves and flame dynamics originally proposed by Markstein [9, 10] has been analytically[11, 12], numerically[13, 14], and experimentally[15-17] studied. A main outcome is that acoustic instabilities are more likely to emerge when the Markstein number is low [8], (e.g rich propane-air flame [18]). Controlling the primary instability in tubes, by using an acoustic source (loudspeaker) or an acoustic damper (sub-millimeter aperture) at the closed end of the tube, allowed to study the Darrieus-Landau intrinsic wrinkling [19, 20] in the linear [21] and non linear [22] regimes.

More recently, accurate studies of the Darrieus-Landau wrinkling have been performed in an experimental device which allows to reduce the flame dynamics to a quasi-bidimensionnal one [18, 23-29]. The two dimensional dynamics has been shown to be similar to that of freely propagating flames [18]. In particular, the front corrugations undergo a complex motion causing permanent fluctuations of the total flame area. Due to the induced fluctuating heat release, one would expect the emergence of acoustic instabilities involving the acoustic modes of the burner in the same way as it does with the tubes. Surprisingly, acoustic instabilities were reported in the experiments solely when the gap was large enough (more than $7 \mathrm{~mm}$ ), or for glass plates sufficiently thick $(19 \mathrm{~mm})[16,30,31]$. For smaller gap or smaller thickness of the glass plates, no oscillating instability was obtained, one possible reason being that viscous losses and acoustic loss at walls are too large to allow pressure fluctuations to excite the acoustic mode of the burner [32]. However, in most of the Hele-Shaw burners, some transient pseudo-periodic oscillations of the flame speed have been observed just after the ignition of the flame of the flame front (see Alexeev et al. [27] Fig. 3 or Jang et al. [26] Fig. 3 or Al Sarraf et al. [18] supplementary material Fig. S2). As remarked by Alexeev et al. [27], these oscillations are not corresponding to the fundamental acoustic mode that is usually observed in tubes, indicating that the coupling with the burner seems to be different.

The purpose of the present paper is to investigate the emergence of velocity and pressure oscillations in Hele-Shaw burners. This phenomenon is acting on the wrinkling of flames and change this way their whole dynamics. We demonstrate that the thermo-acoustic instability usually observed in tubes is limited and also that it can be overcome by another new oscillating instability that emerges from a coupling with the structural modes of the burner walls. In $\S$ II we introduce the apparatus used to tackle this problem. In $\S \mathrm{III}$, we report the diverse oscillating instabilities appearing in the diverse configurations of the Hele-Shaw cell. In $\S \mathrm{IV}$, the structural modes of the burner are studied both analytically and experimentally, and it is shown that these modes are excited during the flame propagation. In $\S \mathrm{V}$ we show that flow oscillations can be induced inside the burner by forcing these structural modes. These oscillations act on the flame in a similar fashion than in the classical thermo-acoustic scenario. Finally, a summary of the results and a short discussion about their potential applications is given in $\S \mathrm{VI}$.

\section{EXPERIMENTAL APPARATUS}

The Hele-Shaw burner studied here is composed of two plates, one $19 \mathrm{~mm}$ thick glass plate and another plate chosen in a set of plates made of either glass or PPMA, with thickness between $5 \mathrm{~mm}$ and $19 \mathrm{~mm}$. The two plates are $500 \mathrm{~mm}$ large and $1500 \mathrm{~mm}$ high, separated by a $5 \mathrm{~mm}$ gap. By taking a different thickness or material for each plate, we expect to reduce the coupling between the plates, and keep the thicker one static to simplify the analysis. The Hele-Shaw burner is oriented vertically, closed at the bottom and on the two sides, and open at the top. A mechanical forcing method is used to study the structural modes of the burner by positioning an electrodynamic vibration exciter (SmartShaker K2004E01) on one plate (see Fig. 1). In order to measure the vibrating response of the plates, several accelerometer are positionned on the plates in order to measure their vibrating response. In addition, electret microphones are inserted in the cell sides at height 0, 30, 60, 90 and $120 \mathrm{~cm}$ to measure pressure fluctuations. In order to initiate the flame propagation, the gas mixture is initially injected by a flow line from the 


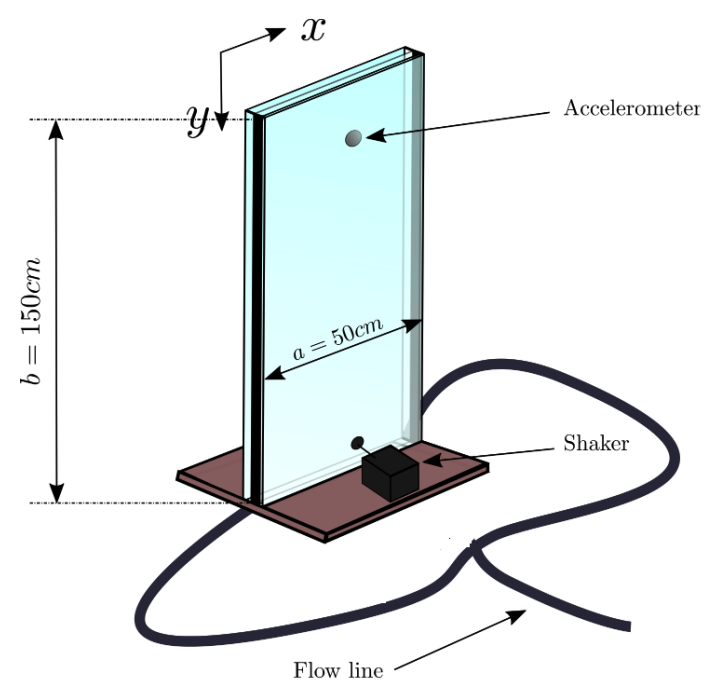

Figure 1. Experimental apparatus: Hele-Shaw burner consisting of two plates (one is made of glass with $19 \mathrm{~mm}$ thickness ) separated by a $5 \mathrm{~mm}$ gap. A shaker can force the plates. Accelerometer probes can be placed on the plates and electret microphones are inserted in the cell side.

bottom of the burner. The flow rate and the equivalence ratio $(\varphi)$ of the combustible mixture are controlled with less than $1 \%$ error thanks to a PC-interface connected to Bronkhorst EL-Flow series mass-flow regulators. The flow of reactive gas is then stopped and the flame is ignited at the top and starts its downward propagation.

\section{SELF INDUCED VIBRO-ACOUSTIC OSCILLATIONS}

We analyze the downward propagation of propane-air flame with equivalence ratios in the range 0.7 to 1.5 with each plates set. This range corresponds to the range where the flame can propagate inside the Hele-Shaw cell of $5 \mathrm{~mm}$ gap without thermal extinction due to heat loss on the walls [18]. Acoustic instability usually appears during propagation in tubes [8]. At the expected frequency, the burner height $\mathrm{E}$ corresponds to the quarter of the acoustic wavelength. The corresponding frequency in our apparatus would be $c / 4 \mathrm{E} \approx 50 \mathrm{~Hz}$. With PMMA plates, such a frequency is not observed, but some low frequency oscillations appear instead. A typical pressure signal recorded during the propagation of a stoichiometric flame is reported on Fig.3(a). The periodogram of this signal is drawn in Fig. 3(b) and indicates the emergence of oscillations around $11 \mathrm{~Hz}$, which is too low to be associated with pure acoustic waves. When two $19 \mathrm{~mm}$ glass plates are used instead, some acoustic oscillations around $50 \mathrm{~Hz}$ appear for equivalence ratios 1.4 and 1.5. In order to figure out which of the acoustic(-like) instability frequency is likely to appear, we report on table I the measured frequency for each set of plates. We observe that the frequency of the pressure oscillations is increasing when using glass plates instead of PMMA plates. The frequency is also increasing with the plate thickness, until classical acoustic instability emerges when both plates are of glass and are $19 \mathrm{~mm}$ thick. These observations provide the evidence that a coupling between the flame propagation and the structure of the burner is at play.

\begin{tabular}{|c|c|c|c|c|c|}
\hline second plate characteristics & $5 \mathrm{~mm}$ PMMA & $8 \mathrm{~mm}$ PMMA & $5 \mathrm{~mm}$ glass & $10 \mathrm{~mm}$ glass & $19 \mathrm{~mm}$ glass \\
\hline main oscillation frequencies $(\mathrm{Hz})$ & 8 & 11 & $63-115$ & 105 & 50 \\
\hline range of unstable equivalence ratio $(\phi)$ & $0.9-1.4$ & $0.9-1.5$ & $1.3-1.4$ & $1.2-1.4$ & $1.2-1.4$ \\
\hline
\end{tabular}

Table I. Frequencies of the self-induced oscillations appearing in a Hele-Shaw cell with a $19 \mathrm{~mm}$ glass plate on one side and one with characteristics reported on the first line on the other. The range of unstable propane-air equivalence ratio is reported for each case.

In order to have a better understanding of the oscillations appearing in the cell we focus on the $5 \mathrm{~mm}$ glass plate configuration. The burner is prepared with two accelerometers on the thin plate to measure plate vibrations in addition to a microphone at the bottom of the cell to measure the pressure fluctuations. One accelerometer is positionned at the top of the plate $(x=250 \mathrm{~mm}, y=0 \mathrm{~mm})$ and the other one at $(x=250 \mathrm{~mm}, y=800 \mathrm{~mm})$. In order to trigger self-induced oscillations, the burner is then filled with a rich $(\varphi=1.4)$ propane-air mixture. The signals measured 

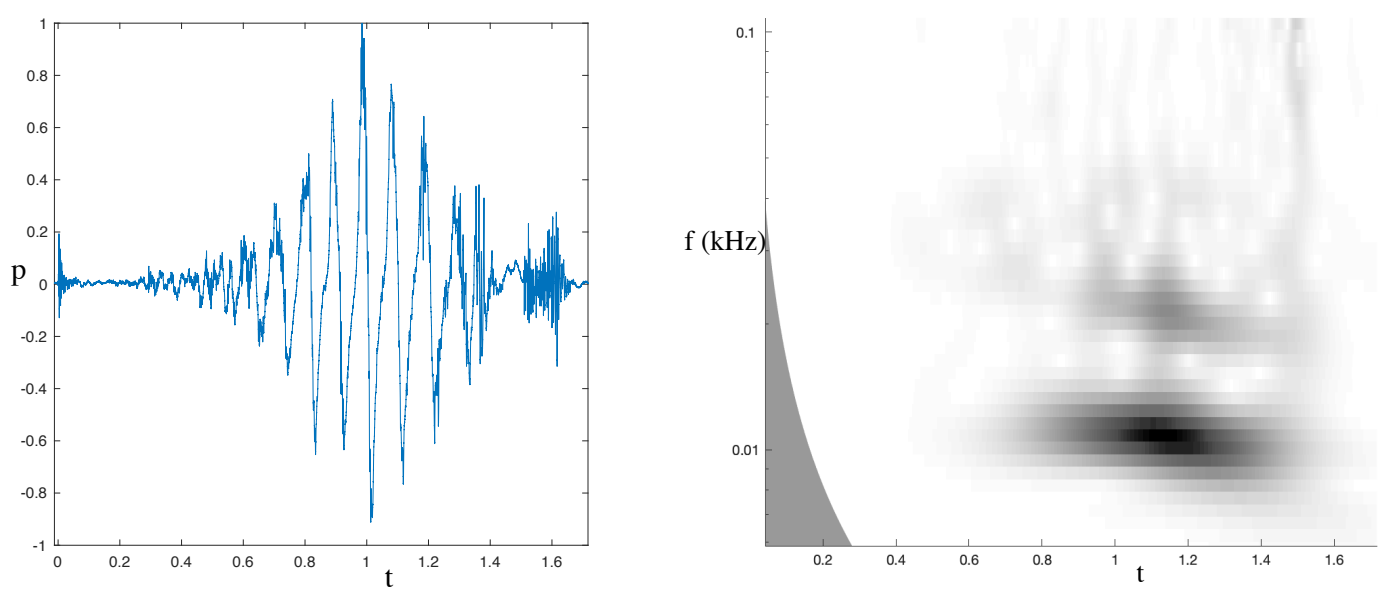

Figure 2. Left: Evolution of the pressure fluctuations during propane-air flame (equivalence ratio 1.2) propagation when the second plate is a PMMA plate of $8 \mathrm{~mm}$ of thickness. Right: associated periodogram exhibiting a $11 \mathrm{~Hz}$ frequency.

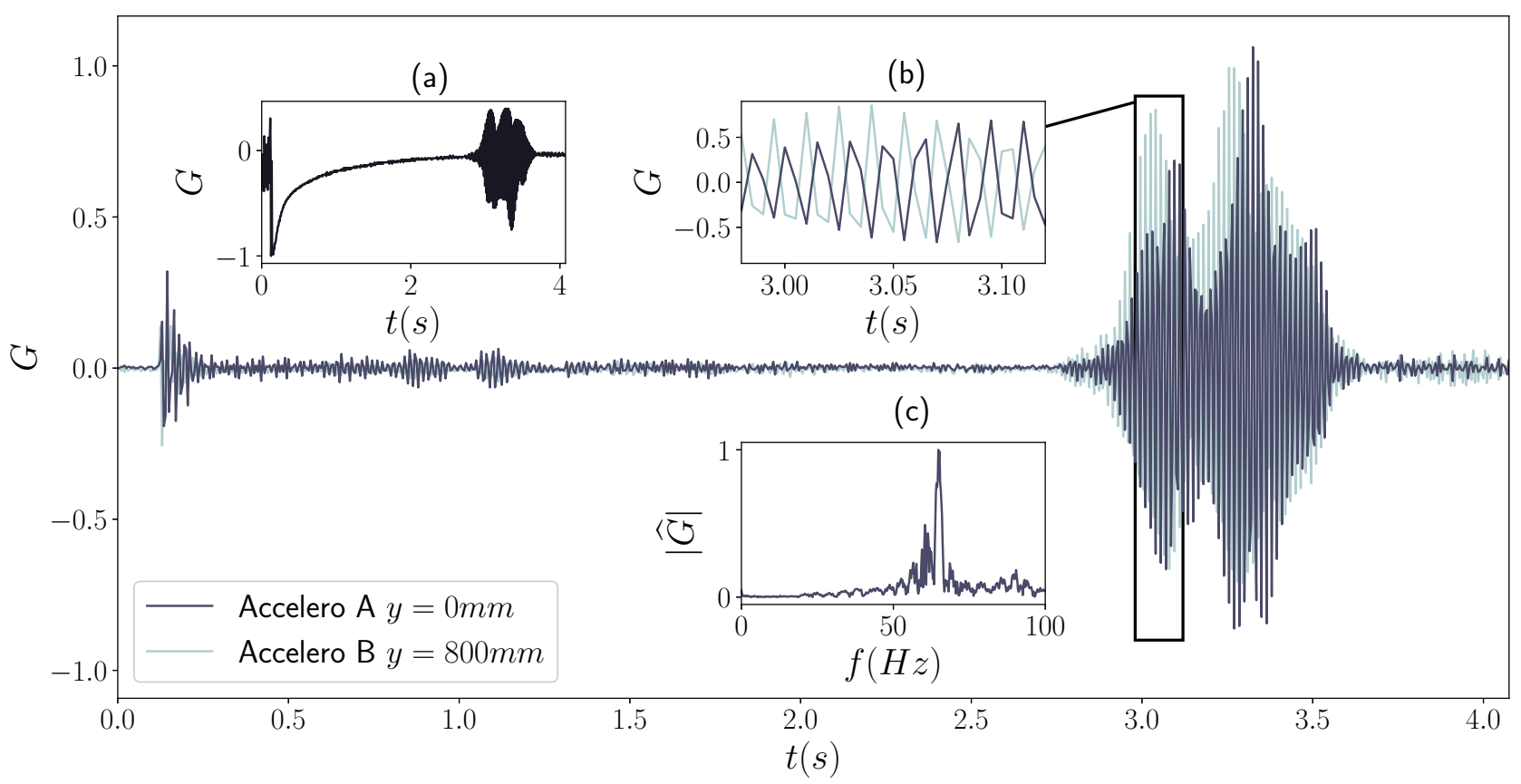

Figure 3. Plate vibrations induced by a propane-air flame $(\varphi=1.4)$ propagating downward with the $5 \mathrm{~mm}$ glass plate configuration. The strong oscillations that emerge when the flame is in the bottom part of the burner are recorded by two accelerometers at two different positions. (a) signal recorded by the microphone at the bottom of the cell. (b) detail in the black rectangle. The signals recorded by the two accelerometers are in phase opposition. (c) Fourier transform of the signal recorded by upper accelerometer. The dominant mode emerges at $f=63 \mathrm{~Hz}$.

by the microphone and the two accelerometers during flame propagation are reported on Fig.3. Both the microphone (Fig.3 (a)) and the accelerometers signals (Fig.3 (b)) exhibit strong oscillations when the flame reaches the second part of the burner. Through Fourier transform (Fig.3 (c)), the dominant mode of these oscillations is clearly visible at $f=63 \mathrm{~Hz}$. Moreover the two accelerometers are in phase opposition. These observations give us indications on the type of deformation undergone by the burner. In the next section, we demonstrate that this deformation correspond actually to an eigen mode of the burner structure. 


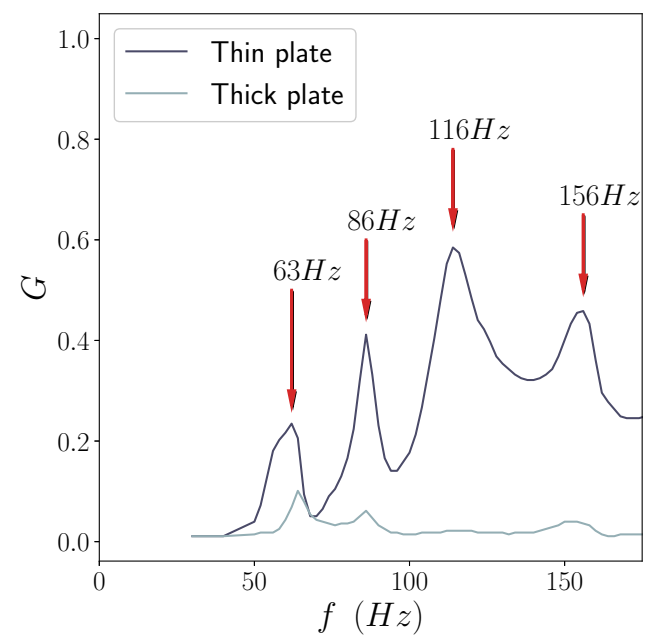

Figure 4. Vibrating accelerations measured by the two probes. The first four resonant frequencies of the thin plate are highlighted by red arrows.

\section{PLATE VIBRATION}

In order to understand the frequencies observed on table I, we plot on Fig. 4 the response of the burner deformation to a vibration excitation when the $5 \mathrm{~mm}$ glass plate is used. As can be seen, in the range $30 \rightarrow 180 \mathrm{~Hz}$, the oscillation amplitude of the $19 \mathrm{~mm}$ thick plate is always at least one order of magnitude smaller than that of the thin plate. Thanks to this observation, we can consider that for this frequency range, the response of the structure can be reduced to the thinner plate one. We identify this way the resonant modes and frequencies of the thin plate, including the previous self induced $63 \mathrm{~Hz}$ oscillations appearing when the rich propane air flame propagates freely inside. This frequency can be approximated theoretically using the Rayleigh method. The detailed calculation is presented hereafter. Following the Kirchhoff plate theory, the free vibrations of an isotropic plate are governed by the biharmonic wave equation [33]:

$$
w_{t t}+\frac{D}{\rho h} \nabla^{4} w=0
$$

where $w(x, y, t)$ is the transverse displacement of the plate of material density $\rho$, Young modulus $E$ and Poisson's ratio $\nu . h$ is the thickness of the plate and $D=E h^{3} /\left(12\left(1-\nu^{2}\right)\right)$ its bending stiffness.

This equation cannot be solved analytically in a general case. However, providing the appropriate boundary conditions the resonant modes of the plate can be approximated using the Rayleigh method [34]. The plate modes for the transverse displacement $w(x, y, t)=W(x, y) \exp (i \omega t)$ are considered as the product of two beam modes $W(x, y)=$ $X(x) Y(y)$. In the present apparatus the plate is considered as simply supported on the bottom side and on the two vertical sides and free on the top side, leading to the following boundary conditions :

$$
\begin{array}{r}
X(x=0)=X(x=a)=Y(y=b)=0 \\
\left.\frac{\partial^{2} X}{\partial x^{2}}\right|_{x=0}=\left.\frac{\partial^{2} X}{\partial x^{2}}\right|_{x=a}=\left.\frac{\partial^{2} Y}{\partial y^{2}}\right|_{y=b}=0 \\
\left.\frac{\partial^{2} Y}{\partial y^{2}}\right|_{y=0}=0,\left.\quad \frac{\partial^{3} Y}{\partial y^{3}}\right|_{y=0}=0
\end{array}
$$

This leads to one linear system for each direction $x$ and $y$ whose eigenvalues (respectively eigenvectors) correspond to the resonant wavenumbers (respectively resonant mode shapes) of the plate in the corresponding direction. The resulting vibration amplitude writes:

$$
W(x, y)=\left(\frac{\sinh \left(\alpha_{2} b\right)}{\sin \left(\alpha_{2} b\right)} \sin \left(\alpha_{2}(y-b)\right)+\sinh \left(\alpha_{2}(y-b)\right)\right) \sin \left(\alpha_{1} x\right)
$$



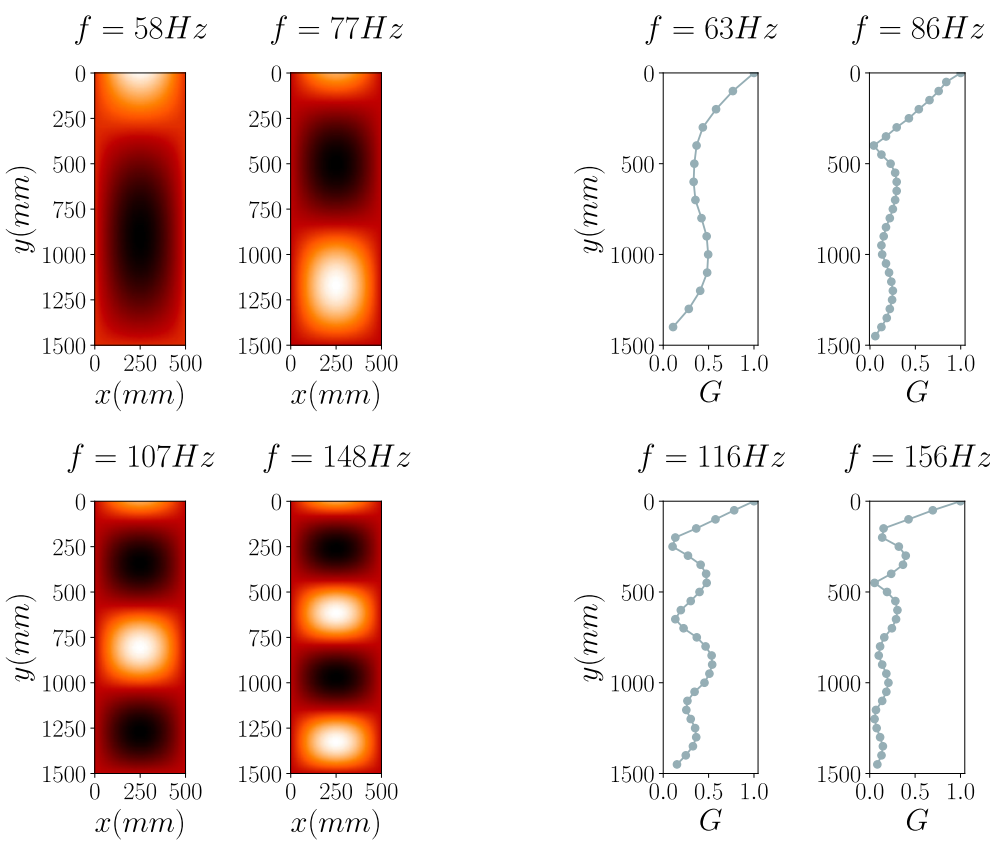

Figure 5. Comparison of theoretical plate mode shapes and frequencies (left) with the experimentally measured ones along the vertical with accelerometers (right).

where $\alpha_{1}=m \pi / a$ is the wavenumber of the $m^{t h}$ resonant mode in the $x$ direction and $\alpha_{2}(n)$ is the wavenumber of the $n^{t h}$ mode in the $y$ direction. $\alpha_{2}(n)$ is the solution of $\tan \left(\alpha_{2} b\right)=\tanh \left(\alpha_{2} b\right)$ which cannot be solved analytically but is well approximated by $\alpha_{2}(n)=(4 n+1) \pi / 4 b$. The approximate pulsation of the resonant mode $(m, n)$ is then given by :

$$
\omega(m, n)=\pi^{2}\left(\left(\frac{m}{a}\right)^{2}+\left(\frac{n+1 / 4}{b}\right)^{2}\right) \sqrt{\frac{D}{\rho h}}
$$

The first four resonant frequencies given by eq.5 for the $5 \mathrm{~mm}$ thickness glass plate $\left(E=69 \mathrm{GPa}, \rho=2500 \mathrm{~kg} \cdot \mathrm{m}^{-3}\right.$, $\nu=0.25)$ are $f(m=1, n=1)=58 \mathrm{~Hz}, f(m=1, n=2)=77 \mathrm{~Hz}, f(m=1, n=3)=107 \mathrm{~Hz}, f(m=1, n=4)=148 \mathrm{~Hz}$ which are in good agreement with the previously experimentally measured resonant frequencies (see Fig. 1(b)). To ensure that these resonant modes correspond to the measures, the shape of the latter is analyzed by measuring the local acceleration $A(x, y)$ along the $x$ and $y$ directions for these four resonant frequencies. The measured relative amplitude $G=A(y) / \max (A(y))$ is then compared to the mode shape given by eq.4 (Fig.5). As predicted analytically, the measured mode in the $x$-direction is $m=1$ for these first four resonant frequencies whereas in the $y$-direction we get $n=1$ for $f=63 \mathrm{~Hz}, n=2$ for $f=86 \mathrm{~Hz}, n=3$ for $f=116 \mathrm{~Hz}, n=4$ for $f=156 \mathrm{~Hz}$ (see comparison on Fig. 5). This ensures that the structural modes identified in Fig.1(b), correspond actually to the eigenmodes of the thinner plate. The slight differences between theoretical and experimental frequencies arise from the connection of the plates to the frame. An increase in the tightening of the plates resulting in a slight increase of the experimental frequency. A similar analysis has been performed with the $8 \mathrm{~mm}$ PMMA plate, and a similar result was obtained, but the first excited mode that emerged was the mode $(m=1, n=0)$ with $10 \mathrm{~Hz}$ frequency. In the next section it is shown that these plate modes may influence the flame propagation by generating flow oscillations in the combustion chamber.

\section{VIBRO-ACOUSTIC COUPLING}

To observe the influence of the plate vibrations on the flame dynamics, we analyze the flame propagation when an eigenmode of the structure is excited. To this end, the shaker frequency is adjusted to one of the eigenfrequencies previously measured. The combustion chamber is filled with a mixture of propane-air at equivalence ratio $\varphi=0.8$. We chose this equivalence ratio in order to be out of the range of self-excitation reported on table I. The flame dynamics is recorded using two high speed cameras (Photron fastcam mini ax-200). The camera frame rate is adjusted to 

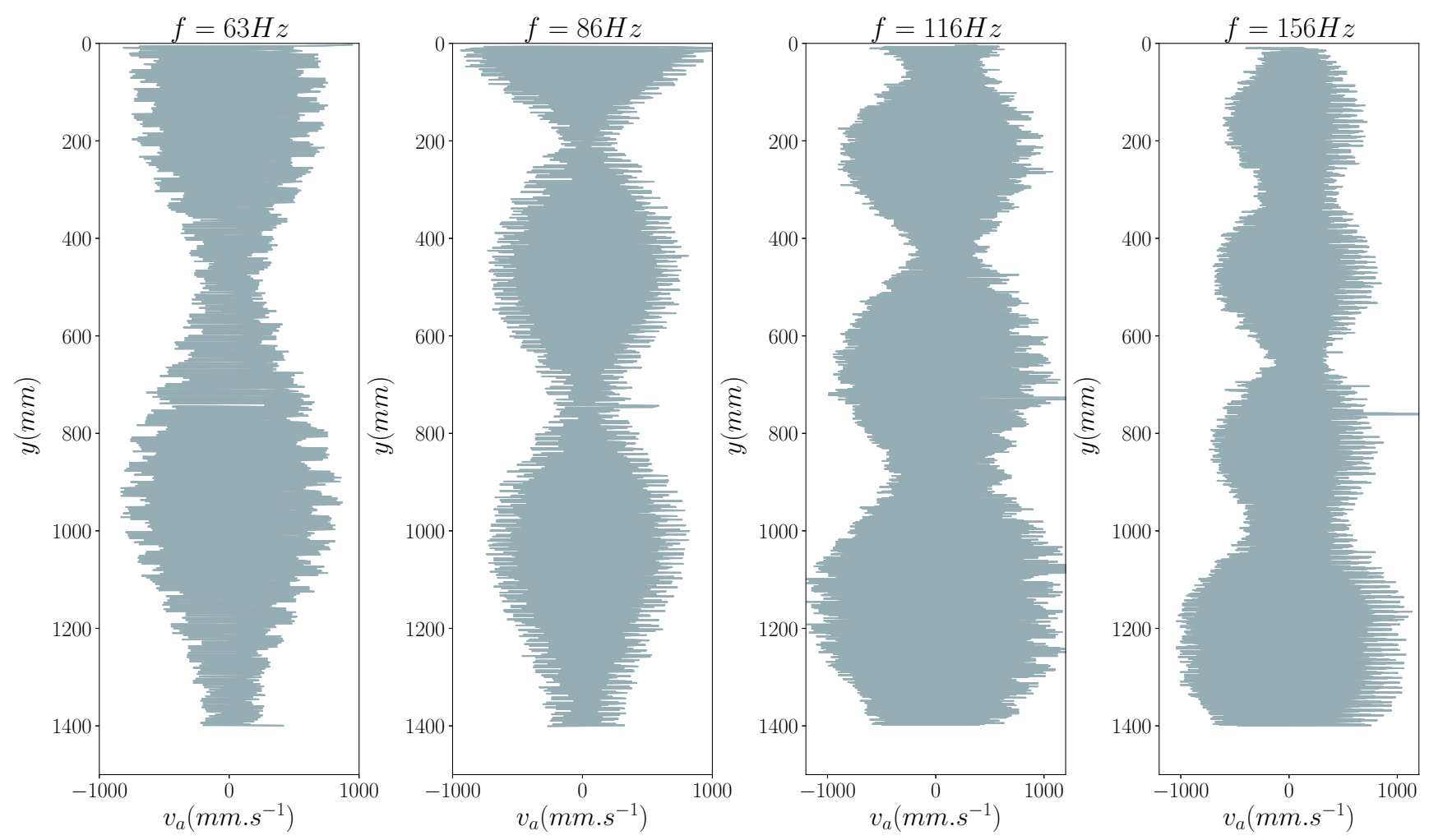

Figure 6. Flow speed oscillations experienced by the flame when one of the eigenfrequencies of the plate is enforced (to compare to Fig.5

be much higher than the oscillation frequency. For each of the four eigenfrequencies identified previously, the flame front coordinates are extracted from these images by way of a python image processing (opensource library scikitimage) with subpixel accuracy (extraction of the bottom part of the front by thresholding methods). From the front coordinates, the mean flame front position at each instant is then computed as $y_{\text {mean }}(t)=1 / a \int_{0}^{a} y(x, t) d x$. Its time derivative corresponds to the oscillating component of the flame velocity:

$$
v_{a}=\frac{d\left(y_{\text {mean }}(t)\right)}{d t}-\frac{y_{\text {mean }}\left(t_{f}\right)-y_{\text {mean }}\left(t_{0}\right)}{t_{f}-t_{0}}
$$

As the flame front is advected by the flow, $v_{a}$ corresponds to the flow oscillations induced by the plate vibrations. Such flow oscillations are then reconstructed along the $y$ axis as it is shown in Fig.6 for the first four plate eigenfrequencies. We observe that the envelop of the oscillations is in agreement with the plate modes. The temporal analysis shows that flow oscillations are in phase with the pressure oscillations measured with electret microphones, and in quadrature with the acceleration of the plates. This is in agreement with a flow induced by the volume variations inside the Hele-Shaw gap: the flow in phase with the velocity of the plate vibrations. In addition, the absence of phase shift between the pressure fluctuations and the flow oscillations indicates that a Poiseuille flow assumption is satisfied.

We can now investigate the influence that these flow oscillations have on the flame shape [11]. As shown by Searby and Rochwerger [8], under periodic flow oscillations, each mode amplitude $\widehat{\Phi}(\tilde{k}, \tilde{t})$ of the flame shape is well described by :

$$
\frac{d^{2} \widehat{\Phi}}{d \tilde{t}^{2}}+2 c \frac{d \widehat{\Phi}}{d \tilde{t}}+\left(\omega_{0}^{2}+a_{1} \cos \left(\tilde{\omega}_{a} \tilde{t}\right)\right) \widehat{\Phi}=0
$$

Where $\tilde{\omega}_{a}$ stands for the non dimensional pulsation of flow velocity fluctuations and $c, \omega_{0}$ and $a_{1}$ are coefficients depending on the non-dimensional wavenumber of the perturbation and on the properties of the combustion reaction. Expression 7 is a damped parametric oscillator equation of eigen pulsation $\omega_{0}$ and damping coefficient $c$. As a consequence, a flame front exposed to periodic flow velocity oscillations is prone to exhibit both parametric restabilization(leading to a flattening of the wrinkles) and parametric resonance (leading to small scale wrinkling) 


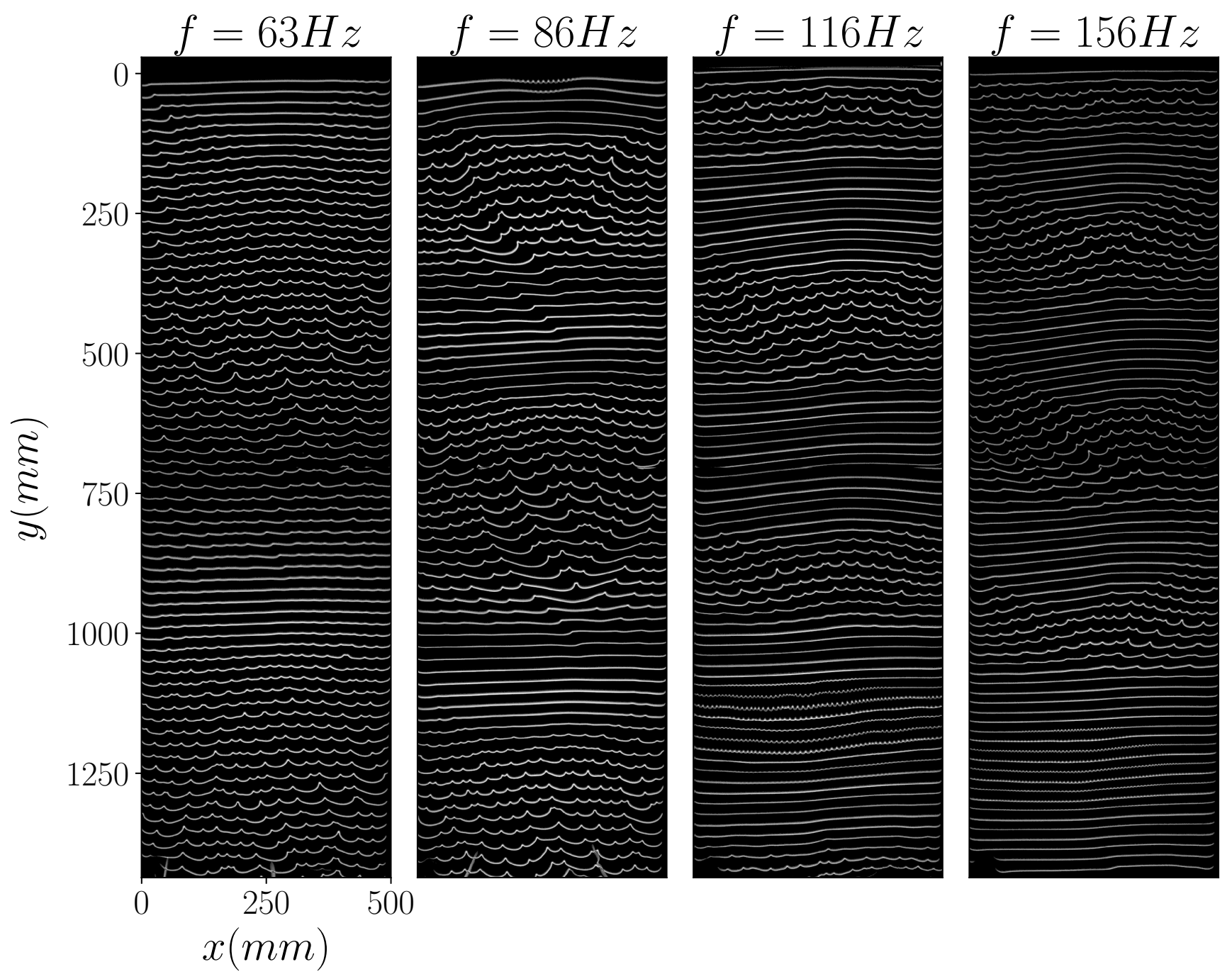

Figure 7. Flame propagation when the plate is forced for the first four resonant frequencies. The front wrinkling undergoes periodic restabilization/destabilization during its propagation. The restabilization (respectively destabilization) zone corresponds to the antinodes (respectively nodes) of the enforced plate modes.

$[8,16,17,35]$. The effect of the variable amplitude of the forcing $v_{a}$ (Fig.6) along the cell on the flame front stability is analyzed by looking at the flame appearance during its propagation (see Fig.7). For all studied forcing frequencies the flame starts its propagation as a flat flame and experiences at some locations the Darrieus-Landau wrinkling, exhibiting the cellular pattern dynamics described in [28]. But during its propagation, the front undergoes periodic restabilization/destabilization in areas whose locations depend on the forcing frequency. Comparing Fig.7 with Fig.6, one can note that along the $y$ axis, the restabilization zones correspond to the antinode zones for the flow velocity oscillations. Moreover, the restabilizations are faster in the center, at $x=250 \mathrm{~mm}$, where the plate vibration and the flow are maximum according to $m=1$. The Searby and Rochwerger [8][12] theory predicts two different wrinkling regimes for flame front advected by flow velocity oscillations. For small $v_{a}$ and sufficient Froude number, a range of unstable wavenumbers is delimited by two cutoff wavenumbers: $k_{g}$ (respectively $k_{c}$ ) where gravity (respectively thermal diffusive) effects are damping the Darrieus-Landau wrinkling. Increasing the forcing intensity $v_{a}$, the range of unstable wavenumbers is reduced. Above the threshold $v_{a}>v_{I}^{*}$, the Darrieus-Landau wrinkling zone is completely suppressed for all wavenumbers. The theory predicts a secondary instability zone which appears when $v_{a}>v_{I I}^{*}$ and is called parametric instability. The two threshold $v_{I}^{*}$ and $v_{I I}^{*}$ depend on the physico-chemical parameters associated with the flame. If for some flames $v_{I I}^{*}>v_{I}^{*}$ and in a range of forcing $v_{I}^{*}<v_{a}<v_{I I}^{*}$ the intrinsically unstable flame undergoes a parametric restabilization. It is exactly what we observe in our experiments, in the antinodes the threshold $v_{I}^{*}$ is reached and the flame remains flat (stable). Then, when the flame propagates further downwards, 


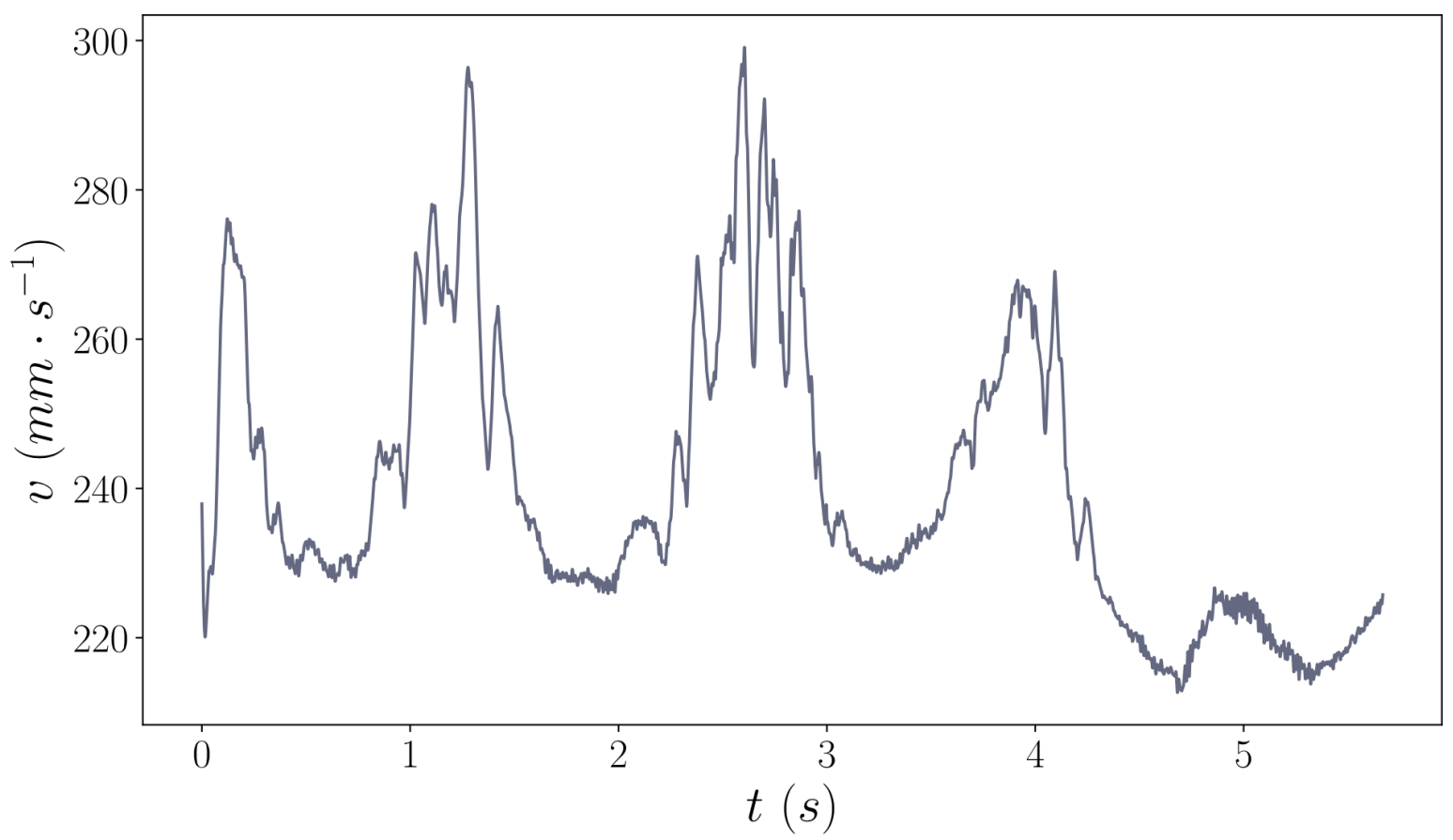

Figure 8. Flame propagation speed when propagating in the cell with forcing at $156 \mathrm{~Hz}$ at play. We can observe that the velocity increases in the zones where Darrieus-Landau wrinking is at play and decreases where the wrinkles are flattened by the parametric restabilization.

the forcing $v_{a}$ decreases until $v_{a}<v_{I}^{*}$ and the flame is once again undergoing Darrieus-Landau wrinkling. This phenomenon is repeated each time the flame crosses an antinode of the modes, which explains the periodic restabilization/destabilization observed in Fig.7. As the flame speed is correlated to the flame shape, we can observe on Fig. 8 that the flame speed is modulated during the propagation in presence of $156 \mathrm{~Hz}$ vibration forcing. The velocity reaches some maxima when the Darrieus-Landau wrinking is at play, in the zone where parametric restabilization is not effective. At the contrary, the velocity decreases when the flame is flattened by the parametric restabilization. This confirms that the vibrations of the plates are efficient to induce flow oscillations in the Hele-Shaw combustion chamber and directly act on the flame flattening and on the flame speed. In addition, when the amplitude of the oscillations is sufficiently high, it is possible to reach parametric destabilization in a similar way as the secondary acoustic instability. When looking at $f=116 \mathrm{~Hz}$ and $f=156 \mathrm{~Hz}$ modes on Fig.7, one can notice a small-scale cellular aspect of the flame, in the last antinode at the bottom of the burner. This small scale wrinkling is oscillating with a period twice the one of the flow oscillations. This is the evidence that it corresponds to the parametric destabilization which arises when $v_{a}>v_{I I}^{*}$. Indeed for $f=116 \mathrm{~Hz}$ and $f=156 \mathrm{~Hz}, v_{a}$ is quite larger in the last antinode than in the others (see Fig.6).

\section{CONCLUSION}

The present study was motivated by the unexplained flame oscillations observed in recent studies on flame dynamics in narrow channels. More precisely, we demonstrated that these oscillations may be caused by structural modes of the burner. These modes have been studied both analytically and experimentally and the obtained results are in agreement. It has been shown that the plate vibration generates flow speed oscillations in the burner, which in turn act on the flame dynamics in a similar fashion to the oscillations induced by thermo-acoustic instability. The important difference here is that the frequency and the mode shape of the oscillations are not ruled by the burner internal geometry like for acoustic modes, but by the structure deformation modes which depends on the material and the whole geometrical characteristics of the burner (thickness, size and assembly). When focusing on the topology of the flame interface, both parametric restabilization and parametric resonance are possible to be forced, as predicted by the Searby and Rochwerger [8] theory. It has been shown that the plate modes may be excited by the flame propagation itself and are certainly responsible for the flame speed oscillations with unexpected frequencies observed 
in recent experiments $[18,26,27]$.

These observations open new ways to study the response of flames wrinkling to flow oscillations. Indeed, the forcing method used in this study is both efficient and easy to set-up, and it allows to study the flame response to time dependent stretch $[36,37$ on a large range of frequencies (expandable by changing the bending stiffness of the plate). Moreover, the distance between the two plates can be easily modified giving opportunity to study the influence of Saffman-Taylor effects on the restabilization threshold $v_{I}^{*}$ and on the parametric destabilization threshold $v_{I I}^{*}$ [32].

We thank 'Agence Nationale de la Recherche' for funding of the ANR 'PDF' ANR-14-CE05-0006, the Excellence Initiative of Aix-Marseille University - A*MIDEX, and Labex MEC, for funding.

[1] Timothy C Lieuwen and Vigor Yang, Combustion Instabilities in Gas Turbine Engines: Operational Experience, Fundamental Mechanisms, and Modeling (American Institute of Aeronautics and Astronautics, 2005).

[2] DJ 1J Harrje and FH Reardon, "Liquid Propellant Rocket Instability," (1972).

[3] Ken H. Yu, Arnaud Trouvé, and John W. Daily, "Low-frequency pressure oscillations in a model ramjet combustor," Journal of Fluid Mechanics 232, 47 (1991).

[4] S. Ducruix, T. Schuller, D. Durox, and S. Candel, "Combustion Dynamics and Instabilities: Elementary Coupling and Driving Mechanisms," Journal of Propulsion and Power 19, 722-734 (2003).

[5] K.C. Schadow and E. Gutmark, "Combustion instability related to vortex shedding in dump combustors and their passive control," Progress in Energy and Combustion Science 18, 117-132 (1992).

[6] KR McManus, Thierry Poinsot, and Sébastien M Candel, "A review of active control of combustion instabilities," Progress in energy and combustion science 19, 1-29 (1993).

[7] J.W.S. Rayleigh, "The theory of sound. Vol. 1," (1896).

[8] G. Searby and D. Rochwerger, "A parametric acoustic instability in premixed flames," Journal of Fluid Mechanics 231, 529 (1991).

[9] G.H. Markstein, "Interaction of Flow Pulsations and Flame Propagation," Journal of the Aeronautical Sciences 18, 428-429 (1951).

[10] G. H. Markstein, Nonsteady Flame Propagation, Vol. 75 (Elsevier, 1963).

[11] P. Pelcé and D. Rochwerger, "Vibratory instability of cellular flames propagating in tubes," Journal of Fluid Mechanics 239, 293 (1992).

[12] Vitaliy Bychkov, "Analytical scalings for flame interaction with sound waves," Physics of Fluids 11, 3168-3173 (1999).

[13] B. Denet and A. Toma, "Numerical Study of Premixed Flames Parametric Acoustic Instability," Combustion Science and Technology 109, 23-33 (1995).

[14] Jorge Yáñez, Mike Kuznetsov, and Reinhard Redlinger, "The acoustic-parametric instability for hydrogen-air mixtures," Combustion and Flame 160, 2009-2016 (2013).

[15] F. Baillot, D. Durox, S. Ducruix, G. Searby, and L. Boyer, "Parametric Response of a Conical Flame to Acoustic Waves," Combustion Science and Technology 142, 91-109 (1999).

[16] R.C Aldredge and N.J Killingsworth, "Experimental evaluation of Markstein-number influence on thermoacoustic instability," Combustion and Flame 137, 178-197 (2004).

[17] Jorge Yanez, Mike Kuznetsov, and Joachim Grune, "Flame instability of lean hydrogen-air mixtures in a smooth openended vertical channel," Combustion and Flame 162, 2830-2839 (2015).

[18] Elias Al Sarraf, Christophe Almarcha, Joël Quinard, Basile Radisson, Bruno Denet, and Pedro Garcia-Ybarra, "Darrieus-Landau instability and Markstein numbers of premixed flames in a Hele-Shaw cell," Proceedings of the Combustion Institute (2018), 10.1016/j.proci.2018.05.030.

[19] G. Darrieus, "Propagation d'un front de flamme," La Technique Moderne 30, 18 (1938).

[20] L.D. Landau, "On the theory of slow combustion," Acta Phys. 19, 77-85 (1944).

[21] C. Clanet and Geoff Searby, "First experimental study of the Darrieus-Landau instability," Physical Review Letters 80, 3867 (1998).

[22] Christophe Almarcha, Bruno Denet, and Joel Quinard, "Premixed flames propagating freely in tubes," Combustion and Flame 162, 1225-1233 (2015).

[23] C. Almarcha, J. Quinard, B. Denet, E. Al-Sarraf, J. M. Laugier, and E. Villermaux, "Experimental two dimensional cellular flames," Physics of Fluids 27, 091110 (2015).

[24] Daniel Fernández-Galisteo, Vadim N. Kurdyumov, and Paul D. Ronney, "Analysis of premixed flame propagation between two closely-spaced parallel plates," Combustion and Flame 190, 133-145 (2018).

[25] Elias Al Sarraf, Christophe Almarcha, Joël Quinard, Basile Radisson, and Bruno Denet, "Quantitative Analysis of Flame Instabilities in a Hele-Shaw Burner," Flow, Turbulence and Combustion 101, 851-868 (2018).

[26] Hye Jin Jang, Gyu Min Jang, and Nam Il Kim, "Unsteady propagation of premixed methane/propane flames in a mesoscale disk burner of variable-gaps," Proceedings of the Combustion Institute (2018), 10.1016/j.proci.2018.06.112.

[27] M. M. Alexeev, O. Yu Semenov, and S. E. Yakush, "Experimental Study on Cellular Premixed Propane Flames in a Narrow Gap between Parallel Plates," Combustion Science and Technology , 1-20 (2018).

[28] C. Almarcha, B. Radisson, E. Al Sarraf, E. Villermaux, B. Denet, and J. Quinard, "Interface dynamics, pole trajectories, 
and cell size statistics," Physical Review E 98 (2018), 10.1103/PhysRevE.98.030202.

[29] J. Sharif, M. Abid, and P.D. Ronney, "Premixed-gas flame propagation in Hele-Shaw cells," (1999).

[30] Daniel Martinez-Ruiz, Fernando Veiga-Lopez, and Mario Sanchez-Sanz, "Vessel confinement contributions to thermoacoustic instabilities of premixed flames," Bulletin of the American Physical Society (2018).

[31] Fernando Veiga-López, Daniel Martínez-Ruiz, Eduardo Fernández-Tarrazo, and Mario Sánchez-Sanz, "Experimental analysis of oscillatory premixed flames in a hele-shaw cell propagating towards a closed end," Combustion and Flame 201, $1-11$ (2019).

[32] R. C. Aldredge, "Saffman-taylor Influence On Flame Propagation In Thermoacoustically Excited Flow," Combustion Science and Technology 177, 53-73 (2004).

[33] AEH Love, The Mathematical Theory of Elasticity (1927).

[34] Arthur W Leissa, Vibration of Plates, Tech. Rep. (Ohio State Univ Columbus, 1969).

[35] G. Searby, "Acoustic Instability in Premixed Flames," Combustion Science and Technology 81, 221-231 (1992).

[36] Guy Joulin, "On The Response of Premixed Flames to Time-Dependent Stretch and Curvature," Combustion Science and Technology 97, 219-229 (1994).

[37] P. Clavin and G. Joulin, "High-frequency response of premixed flames to weak stretch and curvature: A variable-density analysis," Combustion Theory and Modelling 1, 429-446 (1997). 